\title{
Sinergias estratégicas: de la calidad a la infocomunicación. Caso de estudio Clínica Internacional Camilo Cienfuegos
}

DOI: https://doi.org/10.33262/ap.v2i2.26

(c) (i) (우)

Strategic synergies: from quality to infocommunication. Camilo Cienfuegos International Clinic case study

Elizabeth Ramos Sánchez., ${ }^{1}$ Yamilé Ferrán Fernández., ${ }^{2}$ Elizabeth Ramos Sánchez., ${ }^{3}$ \& Delgis Bravo Silva. ${ }^{4}$

\begin{abstract}
.
The ISO 9001 Standard, in its trajectory of more than 30 years, maintains a marked relevance as a quality management model and has evolved towards innovative concepts that are influencing the continuous improvement of the results of organizations. The Camilo Cienfuegos International Clinic (CCIC), has implemented an Integrated Management System (IMS), certified according to the ISO standard, which directly impacts organizational development and its strategic direction. From the ISO methodology and supported by research techniques and matrix tools, the management of information and knowledge become the main axes to analyze the external and internal context and the possible risks and opportunities of the organization to face the challenges of the environment. As well as the

\footnotetext{
${ }^{1}$ Subdirectora de la Clínica Internacional Camilo Cienfuegos, La Habana, Cuba. cirpcc@cicc.cu.

2 Profesora Titular e Investigadora, Facultad de Comunicación, Universidad de La Habana, La Habana, Cuba. yferran@fcom.uh.cu.

${ }^{3}$ Esp. Gestión de Comunicación y Marketing, Clínica Internacional Camilo Cienfuegos, La Habana, Cuba. talia.ramos@cicc.cu

${ }^{4}$ Esp. Gestión de la Calidad, Empresa de servicios a Grupos Electrógenos, La Habana, Cuba.
} 
updating of relevant stakeholders that impact the IMS and the CCIC's ability to provide excellent medical services, from its scientific work, in dialogue with economic policy and commitment to Quality.

Keywords: quality, infocommunication, ISO standard, Camilo Cienfuegos International Clinic.

\section{Resumen.}

La Norma ISO 9001, en su trayectoria de más de 30 años, mantiene una marcada relevancia como modelo de gestión de la calidad y ha ido evolucionando hacia innovadores conceptos que están influyendo en la mejora contínua de los resultados de las organizaciones. La Clínica Internacional Camilo Cienfuegos (CICC), ha implementado un Sistema Integrado de Gestión (SIG), certificado según la norma ISO, que impacta directamente en el desarrollo organizacional y su dirección estratégica. Desde la metodología ISO y apoyado en técnicas de investigación y herramientas matriciales, la gestión de la información y el conocimiento se convierten en los ejes principales para analizar el contexto externo e interno y los posibles riesgos y oportunidades de la organización para enfrentar los retos del entorno. Así como la actualización de las partes interesadas pertinentes que impactan en el SIG y en la capacidad de la CICC para brindar servicios médicos de excelencia, desde su quehacer científico, en diálogo con la política económica y el compromiso con la Calidad.

Palabras claves: calidad, infocomunicación, Norma ISO, Clínica Internacional Camilo Cienfuegos.

\section{Introducción.}

La adopción de un sistema de gestión de la calidad constituye en la actualidad, una decisión estratégica para cualquier organización interesada en optimizar su desempeño global y proporcionarse una base sólida para las iniciativas de desarrollo sostenible. Aunque han existido, desde sus inicios, varios métodos estadísticos de control de la calidad vinculada a los procesos industriales, la industria bélica y otros que posteriormente se fueron diseñando, la fundación de la ISO en 1947 marcó la creación de estándares de uso internacional con la constitución de esta nueva organización integrada por más de 25 países. López (2015).

Si bien el sistema de calidad es autónomo al tiempo que transversal a todos los procesos y subprocesos de la organización, hay consenso en que sobre su articulación han de alinearse otro importante número de subsistemas que como el de información, comunicación y mercadotecnia, son estratégicos en la actual legislación vigente en el país como parte del perfeccionamiento empresarial, a saber Decreto 281 (2007), Reglamento para la Implementación y consolidación de dirección y gestión empresarial estatal. 
Permeados de un contexto mundial convulso, de crisis económica, globalizado, de fuerte movimiento neoliberal, a predominio de gobiernos de derechas, injerencias externas, guerras económicas, bloqueo, retroceso de las relaciones internacionales y de amenaza a la paz mundial, se hace más necesaria la batalla por perfeccionar el Modelo Económico y Social Cubano de Desarrollo Socialista.

En este sentido el Presidente de los Consejos de Estado y de Ministros, Miguel Díaz-Canel, al referirse a la situación financiera del país ha planteado que "la batalla económica sigue siendo la tarea fundamental y también la más compleja" y que "se impone reforzar nuestras estructuras y equipos de dirección y gestión económica con los aportes de especialistas y expertos de las Ciencias Económicas en particular y de otras en general".

En la conceptualización del Modelo Económico y Social Cubano de Desarrollo Socialista, se expone que "se impulsa la creación y avance de las organizaciones empresariales o presupuestadas encargadas de actividades científicas que garantizan la combinación de la investigación y la innovación tecnológica, el incremento rápido, eficiente y eficaz de nuevos productos y servicios, con estándares de calidad reconocidos y una efectiva gestión de comercialización interna y externa", lo que ubica al turismo de salud como un actividad estratégica que, a saber de De la Puente (2015), clasifica como un sector alternativo y dinámico que se encuentra en pleno crecimiento y consolidación con un importante auge en los últimos diez años a nivel mundial.

Los viajes y el turismo contribuyen al 9\% del PIB mundial, con más de $\$ 6$ billones de dólares como promedio anual. Según la Revista Economía de Salud, en el artículo La movilidad de pacientes en el contexto internacional, europeo y español (2012), los viajes en busca de salud han experimentado un cambio de tendencia en los últimos años. El flujo de pacientes desde los países en vías de desarrollo a los países desarrollados, en busca de determinados procedimientos médicos, era lo más habitual hace treinta años.

Hoy día, esta tendencia se está invirtiendo, y no es raro ver pacientes norteamericanos viajando a México o a Costa Rica, en busca de determinados tratamientos médicos. El artículo menciona que las razones principales por las que se ha producido este cambio de tendencia son: la reducción de las listas de espera, la reducción de los costos de los tratamientos, no disponibilidad del procedimiento en el país de origen, calidad percibida, factor que es especialmente relevante en aquellos países que no pueden competir con el costo, gastos propios del viaje, como un aspecto más que contempla el paciente antes de tomar la decisión sobre el destino.

En Cuba la Comercializadora de Servicios Médicos Cubanos, S.A., establece relaciones contractuales directas, para la exportación de servicios de salud. Como parte de su gestión, esta entidad promociona y comercializa, mediante su modalidad de atención a pacientes en Cuba, los servicios especializados de importantes centros e instituciones médicas en el país, 
las cuales marchan a la avanzada tanto en la plataforma tecnológica, investigativa y docente que sustenta sus prestaciones, como la calidad asistencial que las distingue. De hecho, crear la infraestructura (física e institucional) de control de la calidad, así como de normalización, metrología, reglamentación técnica y acreditación, (de manera que garantice el cumplimiento de los requisitos de los mercados globales y un compromiso con la excelencia), es el objetivo del Plan Nacional de la Economía en su eje estratégico No 3. Infraestructura.

Siguiendo esta línea, la Clínica Internacional Camilo Cienfuegos reconoce la necesidad de recertificar su Sistema Integrado de Gestión y continuar perfeccionando su proyección estratégica hacia lo que la propia norma ISO llama, los nuevos modelos de "éxito sostenido". La institución ha recibido importantes premios de calidad conjuntamente con la implementación de un sistema de gestión de calidad basado en norma ISO y Norma Cubana (NC), y que se concibe desde sus inicios, como un Sistema Integrado de Gestión por incluir no solo calidad sino también medio ambiente y posteriormente seguridad y salud, con los que el Sistema Integrado de Gestión de la CICC está certificado por más de 10 años con NC - ISO 9001: 2015, 14001:2015 y NC1800: 2015.

En el tránsito hacia la nueva versión 2015 de la norma, se encuentra que era precisamente el contexto de la organización el aspecto de mayor novedad no solo por ser requisito de obligatorio cumplimiento, sino por todos los acontecimientos que estaban modificando tanto en el macro como en el micro entrono.

De tal suerte, la presente investigación, suscribe los siguientes objetivos:

1. Identificar los ámbitos y procesos en los cuales la Norma ISO prescribe compatibilidad con los procesos de gestión de información y comunicación en las organizaciones cubanas, bajo el encuadre del Perfeccionamiento Empresarial.

2. Analizar estos marcos de actuación en el caso concreto de una entidad de servicio público de salud, con fines de exportación de servicios, la Clínica Internacional Camilo Cienfuegos.

El estudio amén de su novedad, procura situar en coordenada de diálogo y sinergia, especificidades y herramientas de gestión concebidas de forma independientes, con lo cual se asegura la redituabilidad organizacional en la gestión, se minimizan riesgos, se potencian de forma más aguda y holística las oportunidades, lo cual en suma potencia un mayor impacto y posicionamiento de la organización.

\section{Metodología.}

El análisis propuesto se inscribe en la ruta de investigación cualitativa de caso único, respondiendo a métodos generales como análisis-síntesis, inducción, observación, 
interpretación, y a técnicas como observación participante, entrevistas, análisis documental y bibliográfico y grupos focales; por lo que se hizo necesario el empleo de métodos teóricos, empíricos y estadísticos.

Métodos teóricos:

- El enfoque sistémico posibilitó el establecimiento de los aspectos a incluir tanto en el enfoque del macro entorno como en el análisis del micro entorno.

- El análisis-síntesis que facilitó la clasificación de los elementos que integran el Sistema Integrado de Gestión de Calidad y la Norma ISO 9001: 2015, lo que permitió descubrir los requisitos de nueva incorporación.

Métodos empíricos:

- En el análisis documental se revisaron los Manuales de Procedimientos, información documentada del Sistema Integrado de Gestión, documentación legal de la institución, planes de desarrollo, cumplimiento de objetivos de la organización., estadísticas, informes de auditorías internacionales realizadas por la Asociación Española de Normalización y Certificación (AENOR), y los Informes de revisión por la dirección. Este análisis fue muy importante para definir las partes interesadas.

- En la revisión bibliográfica se realizó la exploración, sistematización y valoración de la literatura existente relacionada con el objeto de estudio como publicaciones, páginas web, artículos de la prensa nacional e internacional.

Técnicas:

- Se realizó una lluvia de ideas, con un grupo conformado por responsables de procesos (9) para identificar los aspectos de la norma que eran de nueva incorporación y su importancia para los cambios de denominación, identidad marcaria, y funciones que están ocurriendo en la institución. A partir de la información obtenida por lluvia de ideas se elaboró:

- Diagrama de Causa -Efecto (Ishikawa) en el cual se muestra las causas que conllevan a la necesidad de recertificar el sistema integrado de Gestión. En su confección se utilizó el software Visio 2017.

- Diagrama de Pareto, se utilizó para conocer la frecuencia y peso específico de los Lineamientos de la Política Económica y Social del Partido y la Revolución, en relación al problema de estudio. Para ello se empleó el Software MINITAB versión 17 
- Matriz DAFO: En la Directrices para la aplicación de la Norma ISO 9001:2015 se recomienda que para el análisis del contexto pueden usarse herramientas como DAFO y un enfoque sencillo como lluvia de ideas y preguntas del tipo ¿qué pasaría si...? ISO (2016). A partir de esta recomendación se empleó la técnica DAFO para el diagnóstico de los aspectos positivos y negativos que pueden constituir riesgos oportunidades para la Institución, determinando las debilidades (D), amenazas (A), fortalezas $(\mathrm{F})$ y oportunidades $(\mathrm{O})$. Impacto Cruzado: se analizó las interacciones FO, DO, FA, DA, mediante un valor numérico del uno al cinco, y la preponderancia entre ellas, de modo que se pudiera evaluar el impacto que tienen unas variables sobre otras.

- Matriz direccional: permitió determinar el cuadrante de mayor impacto y determinó el tipo de estrategia a aplicar en la organización: FO -Ofensiva, FA-Defensiva, DOReorientación, DA-Supervivencia.

- Se realizaron análisis grupales con los responsables de los procesos: Gestión de Capital Humano, Gestión Logística y Proceso Asistencial, para identificar las necesidades, expectativas y requisitos de las partes interesadas. Así como el análisis documental de los registros de proveedores, informe de revisión por la dirección, resumen de encuestas de pacientes del año 2018, tal como recomiendan las directrices para la aplicación de ISO 9002:2016 en los métodos de recopilación de información y en los criterios empleados.

- Las partes interesadas pertinentes se clasificaron según matriz influencia - impacto y el registro RG-DP.00.001-003 del SIG.

\section{Resultados.}

\section{Las ISO como encuadre primario.}

Resulta tentador, acaso ilustrativo, apoyarse en el recorrido histórico de la Norma ISO 9001 (publicada por vez primera en 1987), para destacar su relevancia como modelo de gestión, [en permanente y progresiva evolución en el tiempo], cuya efectividad de aplicación da cuenta de más de un millón de organizaciones en todo el mundo.

Esa trayectoria de más de tres décadas es sin duda un precedente a valorar, además de la expectativa que actualmente sigue generando en el entorno empresarial, lo cual confirma el interés demostrado por el avance de la nueva ISO 9001:2015.

Numerosos son los factores que han influido en tal éxito: la propia naturaleza genérica de la norma que le permite ser aplicada en todo tipo de organizaciones; su rol facilitador para las relaciones comerciales entre compañías; asimismo un mercado de certificación que ha servido como punto de encuentro para la confianza de todas las partes interesadas. Gómez (2015) 
No en todas las versiones de ISO 9001 se han incorporado modificaciones de gran calado en los requisitos. La base fundamental para situar el grado de cambio en un escalón u otro, ha sido siempre la voz de los usuarios de la norma y otras partes interesadas. En esta ocasión, la razón de la nueva versión ha sido la necesidad de incorporar o reforzar algunos de los conceptos de gestión que están mejorando los resultados de las organizaciones, y que pueden ser abordados desde la perspectiva de la gestión de la calidad. ISO (2015).

Admiten destacarse como grandes ejes de la nueva norma ISO 9001:2015

- El liderazgo imprescindible de la alta dirección.

- La consideración del contexto como factor estratégico.

- El pensamiento basado en el riesgo, como un elemento dinamizador del enfoque a procesos.

- La gestión del cambio, como valor diferenciador de la organización en un entorno cada vez más exigente.

Tales han sido los aspectos innovadores de norma ISO 9001:2015, coadyuvantes de estandarizaciones posteriores en las ISO 9002:2016 y la ISO 9004:2018 cual documentos que proporcionan las especificaciones técnicas o directrices para la aplicación de la norma ISO 9001:2015. En todos los casos el contexto de la organización establece que:

La comprensión del contexto externo: considera cuestiones que surgen de los entornos legal, tecnológico, competitivo, de mercado, cultural, social, político y económico ya sea internacional, nacional, regional o local. ISO (2015)

La comprensión del contexto interno: considera cuestiones relativas al desempeño global de la organización, recursos, factores operacionales, suministros, valores, cultura, los conocimientos, y la estructura organizacional. ISO (2015). ISO (2016)

Las partes interesadas pertinentes son aquellas personas u organismo que pueden afectar, ser afectada, o percibirse como afectada por una decisión o una actividad de la organización. La institución debería determinar las necesidades y expectativas de partes interesadas pertinentes que pueden tener un impacto en la capacidad de la organización para lograr el éxito sostenido. ISO (2018),

Las partes interesadas pueden ser tanto externas como internas, y puede incluir a los clientes, proveedores, organismos, autoridades legales entre otros según la organización considere.

Entre los múltiples beneficios que le puede aportar un Sistema de Gestión de Calidad a una organización están proporcionar productos o servicios que satisfagan los requisitos del 
cliente y los legales y reglamentarios aplicables, así como facilitar oportunidades de abordar riesgos y oportunidades asociadas con su contexto y objetivos. Lizarzaburu (2016).

Es importante que la Organización determine cuáles de estos factores son pertinentes en la implementación y planificación de un Sistema de Gestión de la Calidad, ya que, de no tomarlos en cuenta, se puede afectar la capacidad para lograr los resultados previstos. Además, esto ayuda a las decisiones estrategias que tome la organización con respecto al Sistema de Gestión de Calidad.

El análisis estratégico de contexto intenta averiguar la posición de la organización respecto al entorno y sus grupos de interés, así como evaluar sus propios recursos y capacidades. Se realiza desde un enfoque "macro" al "micro" y por tanto debe dirigirse tanto al plano sectorial e institucional de toda entidad de cooperación al desarrollo, así como al plano organizacional, es decir en relación a su propia capacidad interna. El análisis de situación y contexto externo e interno servirán de base para la definición y formulación de la estrategia de la organización.

\section{Las fuentes teóricas de referencia.}

A punto de partida de un conjunto de documentos ISO y de las directrices de las propias normas ISO 9001:2015, 9002:2016, 9004:2018, se despliega toda la gestión de información de las tendencias del turismo médico explicadas por De la Puente (2015) y la International Medical Travel Journal (2015), de las proyecciones país contenidas en el Plan Nacional de Desarrollo Económico hasta el 2030, así como las coyunturas políticas, económicas, sociales, climatológicas y demográficas, publicadas en diversas fuentes de información, para llegar a los aspectos positivos y negativos, tendencias y acontecimientos que más impactan sobre la organización.

En particular el contexto interno fue basado en la teoría de Porter (1990) resultando vital para determinar las ventajas competitivas, y el análisis de partes interesadas. Así como los registros del propio SIG y las estadísticas e indicadores hospitalarios.

\section{Trayectoria de una Clínica en constante renovación.}

Desde un enfoque crítico de compromiso, automirada y análisis constante, se suscribe que la Clínica ha transitado por estructuras y encargos diferentes, en sus más de 25 años de existencia. Tal es el caso del año 2017, momento en que, como parte del crecimiento de los servicios médicos cubanos, la institución se diversifica y emprende nuevas rutas que potencian sus fortalezas y cubren las exigencias del crecimiento turístico, urbano y empresarial de la ciudad, por lo que ocurre un cambio de denominación de Centro Internacional de Retinosis Pigmentaria Camilo Cienfuegos, para Clínica Internacional Camilo Cienfuegos. 
Una planeación estratégica actualizada ha llevado a reformular y actualizar el discurso y las metas de la organización.

Figura 1. Diagrama de Causa-Efecto (Ishikawa)
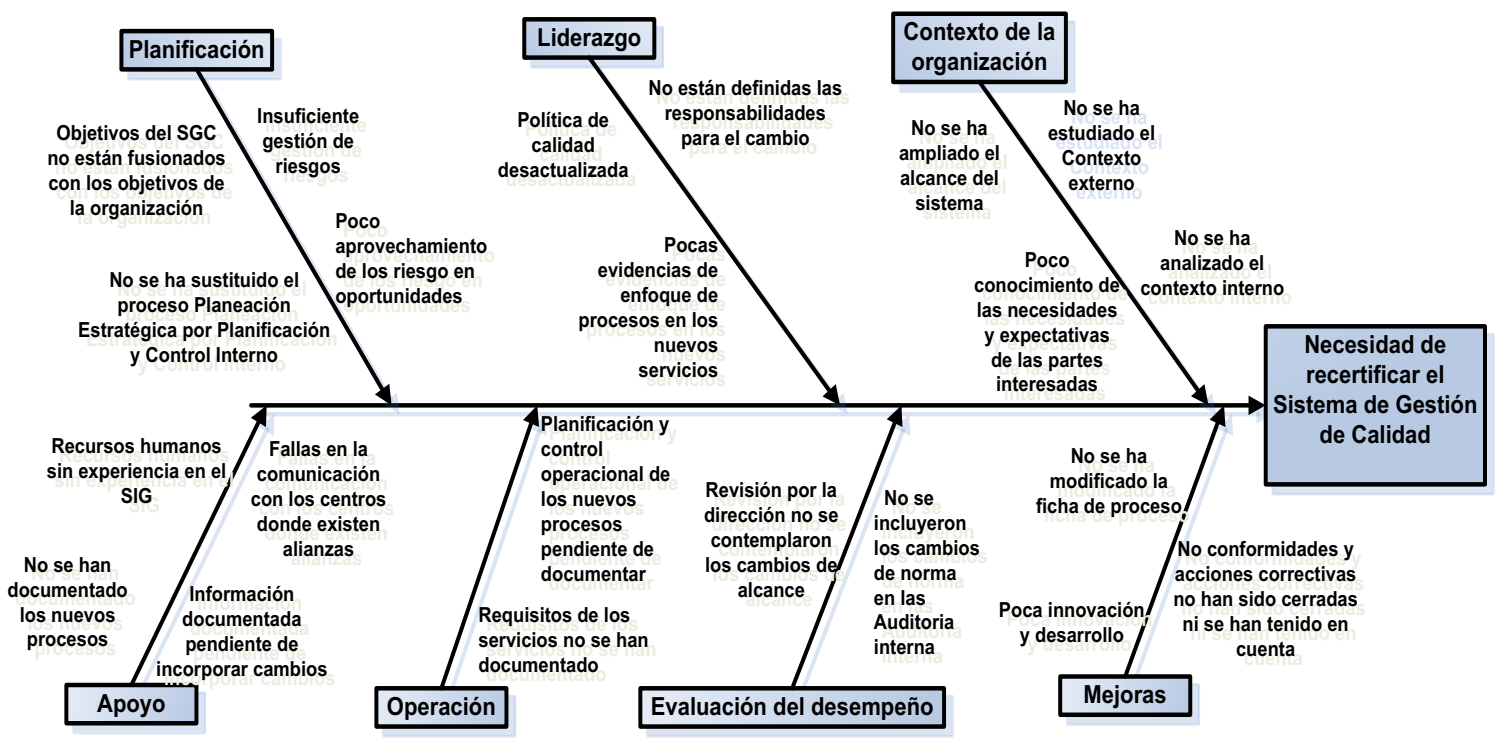

Fuente: Lluvia de ideas apoyada en Plan de transición a ISO 9001:2015 Sistema Integrado de Gestión de la Clínica Internacional Camilo Cienfuegos, año 2018.

En este sentido, la confluencia del pensamiento estratégico entre los líderes de procesos ha sido esencial, para identificar los aspectos de la norma que eran de nueva incorporación y su importancia en los cambios de denominación, identidad marcaria, y funciones que están ocurriendo en el centro. Siendo así, el Diagrama de Ishikawa es útil para develar las causas que conllevan a la necesidad de recertificar el Sistema Integrado de Gestión.

No obstante, a la necesaria renovación, la Clínica mantiene la esencia de sus primeros días fundacionales: articular su labor bajo una filosofía institucional profundamente humanista, que ha sabido actualizar en correspondencia con la misión que cada época ha demandado.

\section{Filosofía institucional:}

En diálogo con las políticas económica, contable-financiera, mercadológica, jurídica, de recursos humanos, científica y de comunicación, la institución privilegia en su quehacer el compromiso con la Calidad.

Uno de los baluartes que caracterizan a esta institución y su fortaleza empresarial es su Sistema Integrado de Gestión, que desde el año 2002 se implementó según la NC ISO 9001:2001, centrado en los servicios asistenciales y aplicaba los ochos principios de Gestión de la Calidad a través del enfoque de sistema para la gestión y del mejoramiento continuo. 
Posteriormente se fueron incorporando a los procesos, aspectos ambientales que influían decisivamente en la materialización de una gestión global segura, ecológica y rentable, según la NC ISO 14001:2004 y por último en el año 2006 integra el Sistema de Gestión de Seguridad y Salud en el Trabajo según la NC 18001:2005.

A la prioridad atribuida a la salud del sujeto en su dimensión biológica y social, se añade la preocupación constante de la administración por los recursos humanos, su permanente capacitación, así como el mejoramiento continuo de las logísticas operacionales con apego preciso a los requerimientos de un SIG, certificado por más de 10 años, con enfoque al cliente y basado en procesos que se acoplan a los modelos NC-ISO 9001:20015, NC-ISO 14001:2015 y NC-18001: 2015 para calidad, medio ambiente y seguridad y salud.

Este Sistema ha elevado los estándares de calidad de sus prestaciones para el alcance de "servicios asistenciales a pacientes ambulatorios y hospitalizados para el tratamiento de la Retinosis Pigmentaria, Cirugía Refractiva por Excimer Laser, Cirugía de Trasplante de Córnea y Cirugía de Catarata".

\section{Futuro avizorado.}

"Seremos la Clínica Internacional polivalente de alta calidad, efíciencia, eficacia y competitividad de la rama del turismo médico mundial, mediante el empleo de la innovación y el desarrollo de las ciencias y la tecnología médica, incluyendo el capital humano, así como otros aseguramientos, equipados con tecnología de punta, con las ventajas de nuestro sistema social y fundamentalmente los valores de nuestros trabajadores".

\section{Análisis contexto externo.}

Para analizar el contexto externo o macro entorno se exponen los aspectos positivos y negativos, políticas, tendencias y acontecimientos que más impactan sobre la organización.

Entorno tecnológico: La persistencia del bloqueo norteamericano es un factor que atenta contra la economía nacional y la gestión de las empresas nacionales, por la necesidad del sector de adquirir equipos de tecnología de punta.

Entorno Económico: En el retroceso de las relaciones entre Cuba y EEUU después de décadas de guerra fría y campañas desestabilizadoras, el fin del bloqueo económico y comercial está aún como una asignatura pendiente.

Contextualización del modelo económico cubano: El modelo económico y social cubano de desarrollo socialista ha establecido los Lineamientos de la política Económica y Social del Partido y la Revolución para el periodo 2016-2021, el Plan Nacional de Desarrollo Económico hasta el 2030. 
Entorno Sociocultural: El país dispone de una gran cantidad de profesionales y técnicos de la salud de elevada calificación.

Entorno Demográfico: Según datos de la $\mathrm{OMS}^{5}$ acerca del envejecimiento y el ciclo de vida a nivel mundial, debido al aumento de la esperanza de vida y a la disminución de la tasa de fecundidad, el número de personas con 60 años o más en todo el mundo se ha duplicado desde 1980, y se prevé que alcance los 2000 millones en el 2050. El envejecimiento de la población mundial trae aparejado un incremento del turismo de salud a nivel mundial, dado el incremento de la demanda de programas de salud, de bienestar y calidad de vida.

Entorno Medioambiental: Cuba se caracteriza por poseer un ambiente sano, tanto desde el punto de vista social como epidemiológico, siendo un destino seguro para el turismo de salud.

Todo esto se fortalece con las normativas medioambientales que deben cumplir todas las unidades de salud pública en cuanto a controles operacionales vinculados al manejo de desechos peligrosos hospitalarios, de residuales de laboratorio clínico, productos químicos, residuales de cocina, control de ruidos, vertederos, entre otros.

Uno de los factores del medioambiente que puede incidir en la gestión de la Clínica es la temporada ciclónica del $1^{\circ}$ de junio al 30 de noviembre.

El Sistema Integrado de Gestión certificado con NC ISO 9001,14001 y 18001 emplean los requisitos legales vinculados a la calidad del servicio, aspectos ambientales y a la Seguridad y Salud en el Trabajo minimizando riesgos, y proporcionando seguridad a pacientes y familiares.

Políticas de apoyo al turismo de salud:

Aunque este tema se debe ajustar al contexto de los diferentes países y no abundan datos al respecto, Fernández (2014) relaciona políticas gubernamentales y empresariales además de las modalidades más significativas:

- Flexibilización de las Políticas de inmigración, como los requisitos para obtener el visado por motivos de salud.

- Incentivos fiscales y otras herramientas de fomento de la inversión en mejora de infraestructuras.

${ }^{5}$ Disponible en www.who.int/features/factfiles/ageing/es/index.html// (publicado en abril del 2012). 
- Apoyo a la creación de entidades que representen los intereses del sector y puedan acometer proyectos asociación de fomento del turismo de salud.

Políticas empresariales:

- Concepción del servicio "turismo de salud": Enfoque hacia la calidad a través de Acreditaciones Internacionales de calidad.

- Modalidades Comerciales: Los canales de comercialización más usados por los principales agentes del Turismo de Salud a nivel internacional son:

- Acuerdos de colaboración con facilitadores de turismo médico.

- Acuerdos de colaboración entre entidades relacionadas con el sector (entre hospitales y hoteles, entre hospitales y compañías aéreas, fundamentalmente)

- Diseño de paquetes turísticos integrando la oferta complementaria.

Alianzas con entidades prescriptoras, como grandes grupos médicos internacionales, grupos hospitalarios de reconocida marca con implantación internacional, así como Sistemas públicos nacionales de salud de terceros países.

Apoyo comunicacional: Diseño de portales web de promoción y comercialización del turismo de salud, normalmente integrados en los portales turísticos de las entidades públicas de promoción turística.

Los portales deben incluir contenidos efectivos, claros y atractivos en las páginas web que permitan a los potenciales clientes obtener información rápida y eficaz y a partir de estas tomar decisiones sin la necesidad de consultar otros destinos. Es importante incluir aspectos de seguridad y calidad.

- Acciones promocionales, tales como asistencia a eventos, ferias, congresos y otros relacionados con el turismo de salud.

- El establecimiento de los ejes psicológicos, así como los argumentos presentes en prácticamente todos los mensajes de Turismo de salud a nivel internacional giran en torno a la calidad y excelencia en el trato al paciente.

\section{Análisis del contexto interno.}

El análisis del contexto interno está basado en las cinco fuerzas del micro entorno de la teoría de Porter, M. (1990) para determinar las ventajas competitivas y el desempeño de la Institución: 
Figura 2. Fuerzas del microentorno.

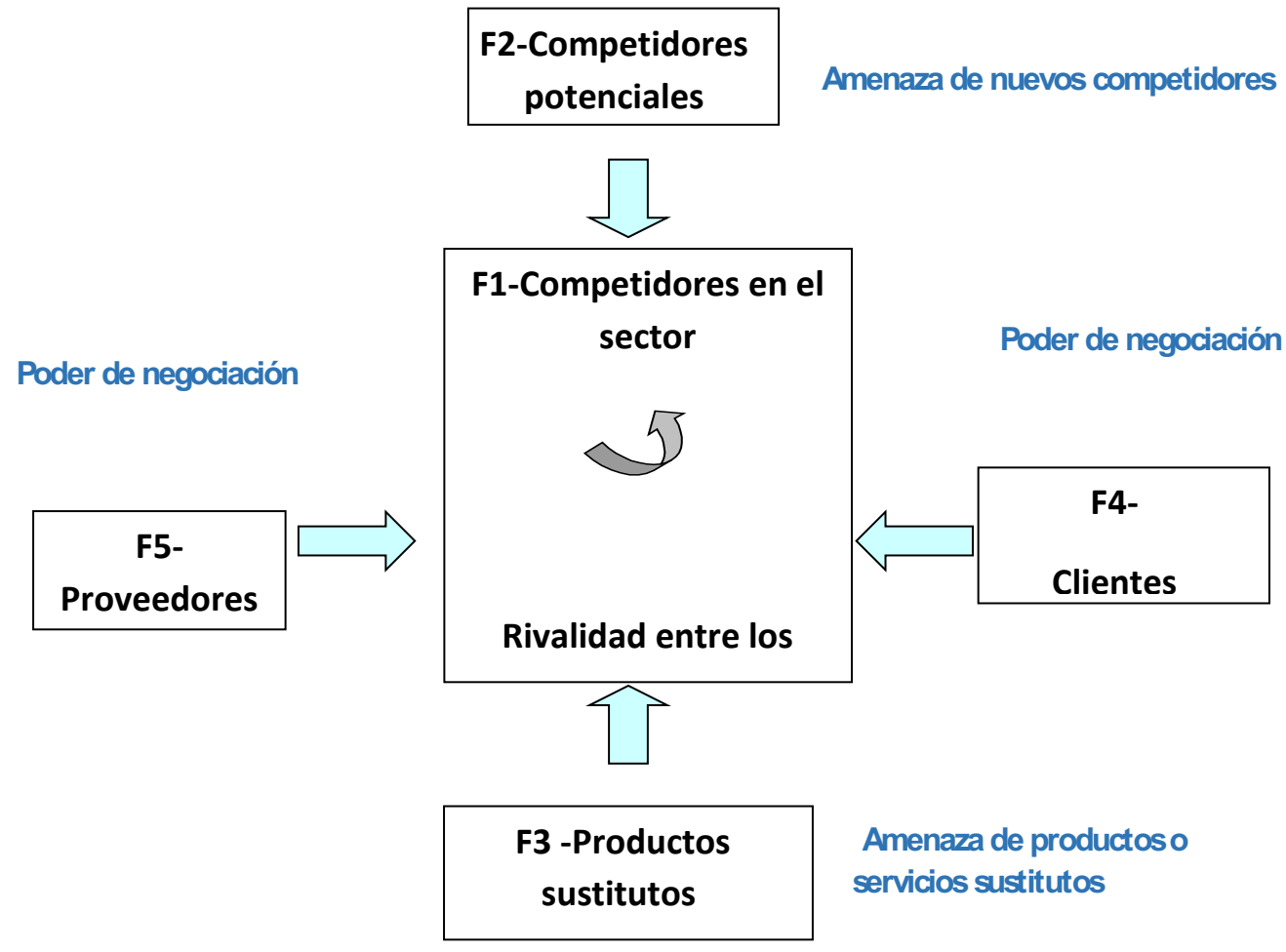

Fuente: Elaboración propia utilizando la matriz de las 5 fuerzas de Porter.

F1: Son las empresas que ofertan producto y servicios similares. A mayor cantidad de competidores más probabilidad de que algunos tomen acciones que provoquen inestabilidad en el sector.

F2: Son empresas que pueden ingresar en el mercado a corto plazo y representar una amenaza, depende de las barreras de entradas al sector y la reacción de las empresas que están operando.

F3: Es la amenaza de sustitución de productos que desempeñan la misma función para el mismo grupo de consumidores basados en una tecnología más avanzada. Si cuentan con precios más atractivos y operan con un mayor rendimiento, representan una mayor amenaza.

F4: Cuando los compradores están más concentrados, influyen en el producto que buscan o sus compras representan un volumen elevado en la empresa puede bajar la rentabilidad de la empresa al aumentar sus exigencias.

F5: El poder negociador de los proveedores aumenta cuando están bien organizados, poseen fuertes recursos y venden productos claves a la empresa. 
En el contexto interno influyen elementos como:

- La competencia

- Amenaza de potenciales entrantes

- Amenaza de productos sustitutos

- Poder negociador de los compradores

- Poder negociador de los proveedores

- Análisis de los mercados

- Análisis de la demanda

- Producto

- Producto clave

- Distribución

- Comunicación

- Procesos

- Personal

- Evidencia Física

Cada una de estas cuestiones relativas al encargo global de la institución intervienen en los productos y servicios de la Clínica, brindados a través de la integración multidisciplinaria. Las rutas de crecimiento de nuevas líneas de productos y servicios son medulares para conformar una cartera de oportunidades que contribuyen a mejorar la salud y el bienestar de los pacientes, generando satisfacción.

Teniendo en cuenta todos los aspectos del entorno y con las técnicas explicadas en la metodología, se elaboraron las matrices DAFO y de Impacto Cruzado.

En el caso de la matriz DAFO, es útil para diagnosticar los aspectos positivos y negativos que pueden constituir riesgos - oportunidades para la institución, determinando las debilidades (D), amenazas (A), fortalezas $(F)$ y oportunidades $(\mathrm{O})$ : 
Figura 3. Matriz DAFO

\begin{tabular}{|c|c|c|c|}
\hline No. & FORTALEZAS & No. & OPORTUNIDADES \\
\hline $\mathrm{F} 1$ & $\begin{array}{l}\text { Sistema Integrado de Gestión, } \\
\text { Certificado por más de } 10 \text { años }\end{array}$ & O1 & $\begin{array}{c}\text { Alto prestigio de la medicina cubana a } \\
\text { nivel internacional }\end{array}$ \\
\hline $\mathrm{F} 2$ & $\begin{array}{l}\text { Posicionamiento y prestigio } \\
\text { empresarial }\end{array}$ & $\mathrm{O} 2$ & $\begin{array}{l}\text { Interés por diversificar la oferta turística y } \\
\text { por el desarrollo del Turismo de salud. } \\
\text { Lineamientos de la Política Económica y } \\
\text { Social del Partido y la Revolución }\end{array}$ \\
\hline $\mathrm{F} 3$ & $\begin{array}{c}\text { Infraestructura hospitalaria, confort, } \\
\text { ubicación, accesibilidad } \\
\text { privilegiada. }\end{array}$ & $\mathrm{O} 3$ & $\begin{array}{l}\text { Realización de diversos eventos } \\
\text { científicos, comerciales. }\end{array}$ \\
\hline $\mathrm{F} 4$ & $\begin{array}{l}\text { Capital humano multidisciplinario } \\
\text { de alto nivel científico de vasta } \\
\text { experiencia, que mantienen su } \\
\text { desarrollo profesional y de alianzas } \\
\text { con otros centros. }\end{array}$ & $\mathrm{O} 4$ & $\begin{array}{l}\text { Posibilidad de efectuar convenios con } \\
\text { ONG. }\end{array}$ \\
\hline F5 & $\begin{array}{l}\text { Tecnología médica avanzada, tanto } \\
\text { para el diagnóstico, priorizando la } \\
\text { adquisición de equipamiento } \\
\text { médico de última generación. }\end{array}$ & O5 & $\begin{array}{l}\text { Alto número de profesionales de la salud } \\
\text { cubana prestando servicios en múltiples } \\
\text { países, que promocionan y fortalecen la } \\
\text { imagen, el prestigio y la calidad de la } \\
\text { medicina cubana }\end{array}$ \\
\hline F6 & $\begin{array}{l}\text { Los niveles de satisfacción de } \\
\text { pacientes y familiares son muy } \\
\text { altos y ha recibido importantes } \\
\text { premios y reconocimientos. }\end{array}$ & & \\
\hline F7 & $\begin{array}{c}\text { Fuerte estructura organizacional } \\
\text { con estabilidad en el proceso de } \\
\text { dirección y Capital relacional } \\
\text { fuerte. }\end{array}$ & & \\
\hline
\end{tabular}

\begin{tabular}{|c|c|c|c|}
\hline No. & DEBILIDADES & No. & $A M E N A Z A S$ \\
\hline D1 & $\begin{array}{l}\text { Insuficientes acciones de } \\
\text { publicidad y Gestión de } \\
\text { comunicación. }\end{array}$ & A1 & $\begin{array}{l}\text { Crisis mundial, desaceleración de las } \\
\text { economías de los países. }\end{array}$ \\
\hline D2 & $\begin{array}{l}\text { Dependencia de más del } 50 \% \text { de un } \\
\text { solo emisor de pacientes. }\end{array}$ & $\mathrm{A} 2$ & $\begin{array}{c}\text { Retroceso de las relaciones internacionales, } \\
\text { enigmas políticos. }\end{array}$ \\
\hline
\end{tabular}


D3

Grupo Electrógeno, fuera de servicio.

D5

Nivel de actividad por debajo de la de la capacidad instalada.

D6

Pocos espacios físicos disponibles, hacinamiento de algunos locales.

D7

Poca producción y eventos científicos.

A3 Bloqueo comercial y económico de EEUU

Insuficientes canales de ventas en los países

A4 y contratos de distribución por parte de la Comercializadora SMC

Fenómenos climatológicos, cambio climático.

Fuente: Criterios expuestos en el trabajo grupal

De la matriz anterior se consideró que:

- Las fortalezas F1, F2, F4, F5 y F6 son aquellas más significativas para el negocio, en tanto, permiten aprovechar las oportunidades que ofrece el entorno y reducir el impacto negativo de las amenazas.

- Las amenazas A1, A2 y A5 son las que ofrecen mayor peligro para la entidad.

- Las debilidades D1, D2, D4, D5 y D6 son las que impiden en mayor grado, aprovechar las oportunidades del entorno y potencian el efecto de las amenazas.

- Las oportunidades $\mathrm{O} 1$ y $\mathrm{O} 2$ son aquellas más importantes, en tanto representan coyunturas de éxito.

Para el análisis de impacto cruzado, se analizó las interacciones FO, DO, FA, DA, y la preponderancia entre ellas, lo que permite evaluar el impacto que tienen unas variables sobre otras, de acuerdo a una escala de valores de uno a cinco:

1- No impacta

2- Impacto bajo

3- Impacto medio

4- Impacto medio alto

5- Impacto alto 
ISSN: 2773-7330

Figura 4. Impacto Cruzado

\begin{tabular}{|c|c|c|c|c|c|c|c|c|c|c|c|c|c|}
\hline & 01 & 02 & 03 & 04 & 05 & $\begin{array}{c}T O \\
T A \\
L\end{array}$ & $A 1$ & $A 2$ & $A 3$ & $A 4$ & $A 5$ & $\begin{array}{c}\text { Sub } \\
\text { TOT } \\
\text { AL }\end{array}$ & $\begin{array}{c}\text { Tot } \\
\text { al }\end{array}$ \\
\hline$F 1$ & 5 & 5 & 4 & 3 & 4 & 21 & 3 & 3 & 1 & 1 & 3 & 11 & 32 \\
\hline$F 2$ & 5 & 5 & 4 & 3 & 4 & 21 & 3 & 3 & 1 & 1 & 3 & 11 & 32 \\
\hline$F 3$ & 4 & 4 & 3 & 2 & 3 & 16 & 2 & 2 & 1 & 1 & 2 & 8 & 24 \\
\hline$F 4$ & 5 & 5 & 4 & 3 & 4 & 21 & 3 & 3 & 1 & 1 & 3 & 11 & 32 \\
\hline$F 5$ & 5 & 5 & 4 & 3 & 4 & 21 & 3 & 3 & 1 & 1 & 3 & 11 & 32 \\
\hline F6 & 5 & 5 & 4 & 3 & 3 & 20 & 3 & 3 & 1 & 1 & 3 & 11 & 31 \\
\hline$F 7$ & 4 & 4 & 3 & 2 & 3 & 16 & 2 & 2 & 1 & 1 & 2 & 8 & 24 \\
\hline $\begin{array}{c}T O T \\
A L\end{array}$ & 33 & 33 & 26 & 19 & 25 & 136 & 19 & 19 & 7 & 7 & 19 & 71 & \\
\hline D1 & 4 & 4 & 3 & 2 & 3 & 16 & 1 & 1 & 2 & 2 & 1 & 7 & 23 \\
\hline D2 & 1 & 1 & 1 & 2 & 1 & 6 & 2 & 2 & 4 & 4 & 2 & 14 & 20 \\
\hline D3 & 3 & 3 & 2 & 1 & 2 & 11 & 1 & 1 & 2 & 2 & 1 & 7 & 18 \\
\hline D4 & 4 & 4 & 3 & 2 & 3 & 16 & 2 & 2 & 1 & 1 & 1 & 7 & 23 \\
\hline D5 & 4 & 4 & 3 & 2 & 3 & 16 & 1 & 1 & 2 & 2 & 1 & 7 & 23 \\
\hline D6 & 4 & 4 & 3 & 2 & 3 & 16 & 2 & 2 & 2 & 2 & 1 & 9 & 25 \\
\hline D7 & 1 & 1 & 1 & 1 & 1 & 5 & 1 & 2 & 3 & 3 & 1 & 10 & 15 \\
\hline $\begin{array}{c}\text { sub } \\
\text { Total }\end{array}$ & 21 & 21 & 16 & 12 & 16 & 86 & 10 & 11 & 16 & 16 & 8 & 61 & \\
\hline total & 54 & 54 & 42 & 31 & 41 & & 29 & 30 & 23 & 23 & 27 & & \\
\hline
\end{tabular}

Fuente: Criterios expuestos en el trabajo grupal 
Al llevar estas interrelaciones a la matriz direccional y como resultante de las matrices aplicadas, la Clínica Internacional Camilo Cienfuegos muestra predominio en el cuadrante fortalezas-oportunidades, seguida del cuadrante debilidades-oportunidades por lo que las estrategias deben ser ofensivas y de reorientación:

Figura 4. Matriz direccional

\begin{tabular}{|c|c|c|}
\cline { 2 - 3 } \multicolumn{1}{c|}{} & OPORTUNIDADES & AMENAZAS \\
\hline \multirow{2}{*}{ FORTALEZAS } & Estrategias Ofensivas & Estrategias Defensivas \\
& $\mathbf{( 1 3 6 )}$ & $(\mathbf{7 1 )}$ \\
\hline \multirow{2}{*}{ DEBILIDADES } & Estrategias de Reorientación & Estrategias de Supervivencia \\
& $(86)$ & $(61)$ \\
\hline
\end{tabular}

Fuente: Criterios expuestos en el trabajo grupal

Las estrategias ofensivas (Maxi-Maxi) - (F-O): son las de mayor impacto. Para generarlas debe apoyarse en qué puede hacer la organización para, apoyándose en sus fortalezas, aprovechar al máximo las oportunidades que se han identificado en el entorno.

Las estrategias de reorientación (Mini-Maxi) - (D-O): Se diseñan para reducir las limitaciones que pueden imponerle a la empresa determinadas debilidades, en el máximo aprovechamiento de oportunidades.

Las estrategias defensivas (Maxi-Mini) - (F-A): Se diseñan para enfrentar los posibles impactos negativos que pueden crearle a la organización las amenazas que se identificaron en el entorno, mediante el máximo aprovechamiento de sus fortalezas.

Las estrategias de supervivencia (Mini-Mini)- (D-A): Son las más "traumáticas". Se generan para reducir el efecto que tienen las debilidades de la organización que puedan agudizar el impacto negativo de amenazas que se ha identificado en el entorno.

\section{De partes a públicos.}

Un analista avezado descubre inmediatamente que el análisis del contexto interno y externo, preestablece un diálogo directo con la concepción de la comunicación interna y externa en la organización, en correspondencia con los interlocutores, funciones y encargos y especificidades del entorno sectorial, empresarial y social cubano, sin desestimar los vínculos más globales.

Si bien el componente partes interesadas, ofrece particular relevancia hacia la gestión de comunicación, y en sentido de ida y vuelta, por cuanto admite nutrirse del Mapa de Públicos que, investigación previa, corresponde abrir y jerarquizar al Manual de comunicación y a la estrategia comunicativa que diseñe la organización. 
Para Calidad, no existe una declaratoria inequívoca de cuáles deben jerarquizarse como partes ni tampoco cuántas, de modo que el discriminador será la propia experiencia que en materia de gestión estratégica tenga la entidad, según la triangulación de su tipología, objeto social, misión/ visión y sobre todo desde la comunicación.

Conforme a ello, desglosar e interpretar la información relativa a las Partes interesadas, conlleva hacer un levantamiento de los Requisitos (necesidades y expectativas de estos públicos estratégicos), los Impactos Positivos y Negativos (Riesgos y Oportunidades), así como las Estrategias, entendiendo estas últimas como las acciones y herramientas que conforman las tácticas a seguir.

Este aspecto al ser una nueva tarea dentro del SIG puede ser ajustado con el tiempo y la experiencia de los responsables de procesos, pero en la selección de las partes interesadas se debe considerar lo siguiente:

- Tener en cuenta aquellos con los que la organización tiene una responsabilidad legal, operativa o fiscal, no olvidando aquellas partes interesadas con las que se tienen establecidos contratos, así como las leyes vigentes o las políticas o prácticas vigentes, como, por ejemplo, administraciones, subcontratados, entre otros.

- Personas que tienen influencia para impedir o impulsar la actividad de la organización, como pueden ser, por ejemplo, ONGs.

- Tener en cuenta aquellas personas y empresas que se encuentren en las zonas donde la organización interactúa ya que pueden ser afectadas por la actividad de la organización y, a su vez, influyen en la buena marcha de esta.

- Clientes

- Proveedores

- Personas que tienen una representación clara de grupos de interés como representantes sindicales.

En el análisis del RG-DP.00.001-003 se puntualizaron algunos aspectos para la mejor compresión de las necesidades y expectativas y se incorpora una nueva parte interesada que corresponde a los competidores categoría que no estaba presente hasta el momento.

La lista de partes interesadas pertinentes de la investigación, es exclusiva para la CICC y los criterios para determinarlas fueron el impacto positivo o negativo de la parte interesada sobre el desempeño de la organización; la capacidad para crear riesgos y oportunidades; y la capacidad para afectar a la organización mediante sus decisiones o actividades. 
En el caso de una organización como el Camilo Cienfuegos se identifican estos apartados como siguen:

Clientes: Pacientes y familiares, en su condición de público estratégico por excelencia. La entidad ha obrado tradicionalmente en el tiempo con un Plan de fidelización, desde el cual el cliente repitente recibe atención personalizada en plaza (con sus altas y bajas en función de la disponibilidad presupuestaria); como es usual en organizaciones de este tipo, el consentimiento informado y la política de información a seguir en cada caso, está investida de un encuadre ético que transita de lo hipocrático a lo cultural/organizacional, y que pasa por el manejo de información veraz en todos aquellos canales informativos que tiene habilitada la Clínica: sitio web, redes sociales, presentación de producto en ferias y eventos científicos, publicaciones indexadas, etc, si bien este segmento precisa o reclama mayor articulación entre la visión de comunicación comercial y la de calidad.

Organismos de Administración Central del Estado OACEs: MINSAP, Comercializadora de Servicios Médicos Cubanos S.A; CITMA; Ministerio de Trabajo y Seguridad Social (MTSS), Contraloría General de la República.

Recursos o Capital humano: Dirección y trabajadores; colaboradores (personal bajo contratas puntuales, asesores, consultores, etc), estimado tradicionalmente como Público interno.

Proveedores: Instituciones de salud, aliadas; obliga a actualizar y depurar el listado de registro de contratos.

Competidores: Clínicas de la región, aquellas que constituyen referente directo para los pacientes con los cuales opera la entidad, Salas de atención médica internacional.

Comunidad: Conforme a la tendencia internacional el ámbito comunitario adquiere en las prácticas de gestión nacional cada vez mayor fuerza; en el caso de la institución Camilo Cienfuegos hay mucho más precedente orgánico desde el Sistema de Gestión Integrado, relativo al componente medioambiental, en tanto se opera gestionando los residuales líquidos; los desechos sólidos, por el cuidado frente a la contaminación por ruidos, los no derrames, el aumento del uso de productos biodegradables, iluminación externa, entre tantos elementos que tributan a la calidad de vida del entorno físico donde se ancla la institución, siendo aún poco documentado este acápite desde la gestión estratégica de la comunicación.

Se concede a los clientes, instituciones de salud aliadas y proveedores las partes interesadas de mayor impacto para la CICC y se incorpora a los competidores como partes interesadas pertinentes teniendo en cuenta que estas son las que más se han diversificado según los cambios del entorno y que impactan en el cumplimiento de la nueva misión de la organización. 
En este contexto, las autoras se atreven a conjeturar que si bien todas las partes prescritas por calidad interesan a los susbsistemas de información y comunicación, no necesariamente todas las partes de interés para una eficiente gestión infocomunicativa interesan a calidad, al menos hasta donde la praxis nacional remite, así pues medios de comunicación, prensa y prescriptores, que siempre constituyen públicos estratégicos para la gestión de comunicación no suelen ser tomados como referentes útiles en la ISO, y en la apropiación del SIG.

\section{Conclusiones.}

- El análisis del contexto externo e interno posicionan a la Clínica Internacional Camilo Cienfuegos en ofensiva para la proyección estratégica: predomina en cuadrante fortalezas - oportunidades y debe apoyarse en sus fortalezas así como aprovechar al máximo las oportunidades del entorno. Está también sujeta a reorientación, ya que desde debilidades - oportunidades, se hace necesario reducir las limitaciones que pueden imponerle las debilidades para aprovechar al máximo las oportunidades, lo que fortalecerá a la CICC y su Sistema Integrado de Gestión.

- La actualización de las partes interesadas pertinentes impacta en el SIG y en la capacidad de la institución para proveer atención médica internacional y otros servicios hospitalarios de alta calidad.

- Se constatan diversos puntos de vínculo que a nivel metodológico y por contenido se verifican entre el sistema de calidad, según Norma ISO y los procedimientos e intereses de los subsistemas de información y comunicación, con lo cual se instituye este entrecruzamiento como necesario, tanto a nivel procedimental como filosofía de la organización, a fin de alinear procesos, eliminar reiteraciones y duplicidades innecesarias; estimular una gestión documental más asertiva; otorgar mayor sinergia y cientificidad a la labor de procesos y vínculos de la organización, así como favorecer la toma de decisiones y la proyección estratégica para una administración pública exitosa y sostenible, al tiempo coadyuve al posicionamiento de la entidad en sentido cada vez más sistémico y holístico.

\section{Referencias bibliográficas.}

Análisis del contexto. http://www.metoder.nu/cgibin/met.cgi?d=s\&w =2024 consultado el 26 de marzo de 2018

De la Puente, M. Dinámica del turismo de salud internacional: una aproximación cuantitativa Disponible en: http://www.docplayer.es/3402479-Dinamica-del-turismo-desalud.Acceso 22 de julio de 2015 
Díaz- Canel, M. Discurso de clausura del segundo periodo de la IX Legislatura de la ANPP. Palacio de Convenciones, 22 de diciembre 2018. http://www.granma.cu/discursosde-diaz-canel.co Acceso 13 de febrero 2019.

Fernández, C. Multisectorialidad, epicentro para estrategias comerciales y comunicacionales. Trabajo de Diploma. Universidad de la Habana.

Folleto Actualización de los Lineamientos de la Política Económica y Social del Partido y la Revolución para el periodo 2016-2021

Gómez JA. Guía para la aplicación de UNE - EN ISO 9001:2015. Edic. AENOR, 2015

ISO. Sistema de Gestión de la Calidad - Requisitos de la Norma ISO 9001:2015

ISO. Sistema de Gestión de la Calidad, Directrices para la aplicación de la Norma ISO 9001:2015 http://www.ISO.org/tc176/ISO9001.

ISO. Sistema de Gestión de la Calidad, Directrices para la aplicación de la Norma ISO 9001:2015. Especificación técnica ISO 9002:2016. 2016.

ISO. Gestión de la Calidad - Calidad de una organización -orientaciones para lograr el éxito sostenido ISO 9004:2018. 2018 4ta edición

Lizarzaburu. La gestión de la calidad en Perú: un estudio de la Norma ISO 9001. 2016.

López P. Novedades ISO 9001:2015. Editorial Fundación Continental, Madrid, 2015. http://repositoriy.unilibre.edu.co consultado 1 de febrero de 2019

McKinsey \& Company 2010. Inspired Wellness. Citado por: Forestieri KT. Determinación del turismo medico como opción de desarrollo económico del sector salud en Colombia. Universidad EAN. Monografía. 2012. Disponible en: repository.ean.edu.co/bitstream/handle/.../ForestieriKelly2012.pdf? Acceso en: 12 de julio de 2014

Medical Tourism Association. Medical Tourism Magazine, February 2008. Disponible en: http://www.medicaltourismmag.com/the-good-the-bad-and-the-ugly/

OMS. Datos interesantes acerca del envejecimiento. Disponible en: http://www.who.int/ageing/about/facts/es Acceso en: 10 de agosto de 2015

Porter, M. Ventaja Competitiva: Creación y sostenimiento de un desempeño superior. México: Continental, c11. 1990

Revista Economía de Salud, La movilidad de pacientes en el contexto internacional, europeo y español (2012). 
Para citar el artículo indexado

Ramos Sánchez, E., Ferrán Fernández, Y., Ramos Sánchez, E., \& Bravo Silva, D. (2020). Sinergias estratégicas: de la calidad a la infocomunicación. Caso de estudio Clínica Internacional Camilo Cienfuegos. AlfaPublicaciones, 2(2), 6-28. https://doi.org/10.33262/ap.v2i2.26

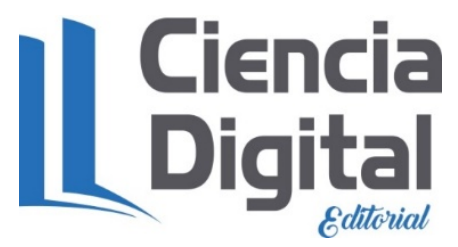

El artículo que se publica es de exclusiva responsabilidad de los autores y no necesariamente reflejan el pensamiento de la Revista Alpha Publicaciones.

El artículo queda en propiedad de la revista y, por tanto, su publicación parcial y/o total en otro medio tiene que ser autorizado por el director de la Revista Alpha Publicaciones.
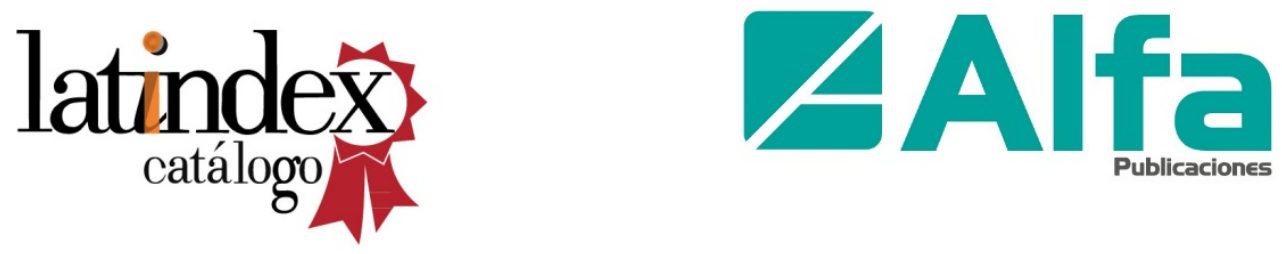